\title{
A Systematic Review of the Impact of Patient-Physician Non-English Language Concordance on Quality of Care and Outcomes
}

\author{
Lisa Diamond, $M D \mathrm{MPH}^{7}$, Karen Izquierdo, $\mathrm{BS}^{2}$, Dana Canfield, $\mathrm{MD}^{3}$, Konstantina Matsoukas, MLIS , \\ and Francesca Gany, MD MS
}

'Memorial Sloan Kettering Cancer Center, New York, NY, USA; ${ }^{2}$ Macaulay Honors College at Hunter College, New York, NY, USA; ${ }^{3}$ University of Utah School of Medicine, Salt Lake City, UT, USA.

BACKGROUND: Approximately 25 million people in the USA are limited English proficient (LEP). When LEP patients receive care from physicians who are truly language concordant, some evidence show that language disparities are reduced, but others demonstrate worse outcomes. We conducted a systematic review of the literature to compare the impact of language-concordant care for LEP patients with that of other interventions, including professional and ad hoc interpreters.

METHODS: Data was collected through a systematic review of the literature using PubMed, PsycINFO, Web of Science, Cochrane Library, and EMBASE in October 2017. The literature search strategy had three main components, which were immigrant/minority status, language barrier/proficiency, and healthcare provider/ patient relationship. The quality of the articles was appraised using the Downs and Black checklist.

RESULTS: The 33 studies were grouped by the outcome measure studied, including quality of care (subdivided into primary care, diabetes, pain management, cancer, and inpatient), satisfaction with care/communication, medical understanding, and mental health. Of the 33, 4 $(6.9 \%)$ were randomized controlled trials and the remaining $29(87.9 \%)$ were cross-sectional studies. Seventy-six percent $(25 / 33)$ of the studies demonstrated that at least one of the outcomes assessed was better for patients receiving language-concordant care, while 15\% (5/33) of studies demonstrated no difference in outcomes, and $9 \%$ $(3 / 33)$ studies demonstrated worse outcomes in patients receiving language-concordant care.

DISCUSSION: The findings of this review indicate that, in the majority of situations, language-concordant care improves outcomes. Although most studies included were of good quality, none provided a standardized assessment of provider language skills. To systematically evaluate the impact of truly language-concordant care on outcomes and draw meaningful conclusions, future studies must include an assessment of clinician language proficiency. Language-concordant care offers

Electronic supplementary material The online version of this article (https://doi.org/10.1007/s11606-019-04847-5) contains supplementary material, which is available to authorized users.

Received April 26, 2018

Revised November 8, 2018

Accepted January 11, 2019

Published online May 30, 2019 an important way for physicians to meet the unique needs of their LEP patients.

KEYWORDS: communication barriers; physician-patient communication; quality of care; language access.

J Gen Intern Med 34(8):1591-606

DOI: $10.1007 / \mathrm{s} 11606-019-04847-5$

(c) Society of General Internal Medicine 2019

\section{INTRODUCTION}

Approximately one in five people in the USA speaks a language other than English at home ${ }^{1}$ with Spanish, Chinese, French, Tagalog, and Vietnamese being the most common ones. ${ }^{2}$ Among these 58 million people, 25 million report having limited English proficiency (LEP). ${ }^{1}$ These individuals have difficulty speaking, reading, writing, or understanding English, ${ }^{3}$ which presents significant obstacles to accessing and receiving care in an English-dominant healthcare system. Due to language barriers, LEP patients have worse healthcare quality and outcomes ${ }^{4-7}$ and decreased access to preventive services $^{8,9}$ and cancer screening. ${ }^{10-13}$ Although federal regulations require healthcare organizations to provide trained interpreters for LEP patients, many hospitals and clinicians are non-compliant with these regulations, leading to persistent inequities in care. ${ }^{14}$ Language concordance, when a physician is fluent in a patient's preferred language, offers a way of reducing these disparities.

Health disparities due to language barriers are reduced when care is provided by language-concordant clinicians. For example, studies demonstrate that LEP patients with language-concordant physicians report receiving more education about their care, have fewer unasked questions, and have better medication adherence and fewer emergency room visits. ${ }^{15-17}$ When a clinician and an LEP patient communicate in a language in which only one of them is fluent, this partial language concordance can further obfuscate clinical interactions by leading patients and providers to believe they understand each other, thus contributing directly and indirectly to medical errors and poor outcomes. ${ }^{14,18}$ While communicating in a patient's native language may build rapport, non-fluent 
clinicians must know when to call an interpreter ${ }^{19}$ and have an accurate gauge of their own limitations. ${ }^{14,}{ }^{20}$ Patient satisfaction with care is higher, adherence to medications is better, and patients have fewer unanswered questions about their care when language-concordant care is provided. ${ }^{15,16,21}$ However, there is also evidence that language concordance is associated with lower cancer screening rates for patients with LEP. ${ }^{17,} 22$ Given these divergent findings, it is important to determine the overall impact of language concordance in clinical settings.

The purpose of this systematic review was to synthesize the data on the impact of language-concordant care for LEP patients compared to other interventions to improve language access, including professional interpreters and untrained $\mathrm{ad}$ hoc) interpreters. We hypothesized that language-concordant care would be beneficial to LEP patients. Our goal was to create a comprehensive picture of the relationship between language concordance and quality of care for patients with LEP.

\section{METHODS}

\section{Literature Search and Data Sources}

We searched 5 databases for this systematic review: PubMed (NLM, 1945 to October 2017), PsycINFO (OVID, 1806 to October 2017), Web of Science (Thomson Reuters, 1945 to October 2017), Cochrane Library (Wiley, 1966 to October 2017), and EMBASE (Elsevier, 1966 to October 2017). Two authors (LCD and KM, a research librarian) developed strategies to find relevant articles. Online Appendix 1 provides detailed search strategies for each database. We did not apply limits to date or article language. The Endnote (Thomson Reuters) bibliographic citation management program was used to manage the citations and remove duplicates.

\section{Data Synthesis and Analysis}

A systematic title and abstract review was conducted by two authors (KI and LD) using the Population, Intervention, Comparison, and Outcome (PICO) framework. ${ }^{23}$ Qualitative articles that systematically gathered data on language-concordant interventions were also eligible for inclusion. Articles were eliminated without further review if they did not focus specifically on the impact of language-concordant care and clinical outcomes. Because of heterogeneity in the methods and outcomes, we pooled our results using qualitative rather than quantitative approaches.

\section{Data Abstraction}

Two authors abstracted data from the remaining 33 articles independently (LD and KI or DC). We abstracted 16 items from each article: study locations, sample sizes, type of clinical outcomes studied, participants' ages (including range, mean, and standard deviation if provided), participants' race and/or ethnicity, languages interpreted, type of patient navigators, study designs, recruitment methods, facility types, comparison groups, and outcomes and results/major findings. One author (LD) reviewed all abstractions and registered any discrepancy between authors. These discrepancies were resolved by consensus. For studies with more than one outcome, we categorized outcomes based on the best result (e.g., if one outcome was better for patients with language-concordant physicians and another showed no difference, we categorized the study as demonstrating better outcomes for languageconcordant care).

\section{RESULTS}

\section{Study Selection}

Our search yielded 8618 citations. After adding 5 articles identified from other sources and removing duplicates and exclusion of articles that were not relevant, 126 articles remained for full-text review. Of these, 92 were excluded yielding 34 articles. During full-text review, one additional article was eliminated because it was a descriptive study of a language concordance intervention for which there were no patient outcomes described. ${ }^{24} \mathrm{~A}$ total of 33 articles were included in our qualitative analysis. Figure 1 shows the PRISMA flow diagram. ${ }^{25}$

\section{Quality Appraisal}

We systematically appraised the quality of all articles using the Downs and Black checklist. ${ }^{26-28}$ For this review, the modified Downs and Black was used. ${ }^{29}$ Online Appendix 2 shows the Downs and Black checklist components for each article. To assess the quality of the language services provided, the authors also abstracted information to describe any language proficiency testing clinicians received, if documented in the study. The quality of the articles overall was very good, with some variation. This was due, in part, to incomplete information from some of the included articles.

\section{Study Characteristics}

Of the 33 articles, 4 (6.9\%) were randomized controlled trials and the remaining $29(87.9 \%)$ were cohort studies. The 33 studies were grouped by the outcome measure studied, including quality of care (further subdivided into primary care, diabetes care, pain management, cancer, and hospital setting), satisfaction with care/communication, medical understanding, and mental health, as shown in Table 1. The USA was the setting of $94 \%$ of the studies (31/33). Seventy-six percent (25/ 33) focused on interventions for Spanish-speaking patients and 30\% (10/33) for speakers of Asian languages.

\section{Language Concordance Outcomes}

Seventy-six percent (25/33) of the studies demonstrated that at least one of the outcomes assessed was better for patients 


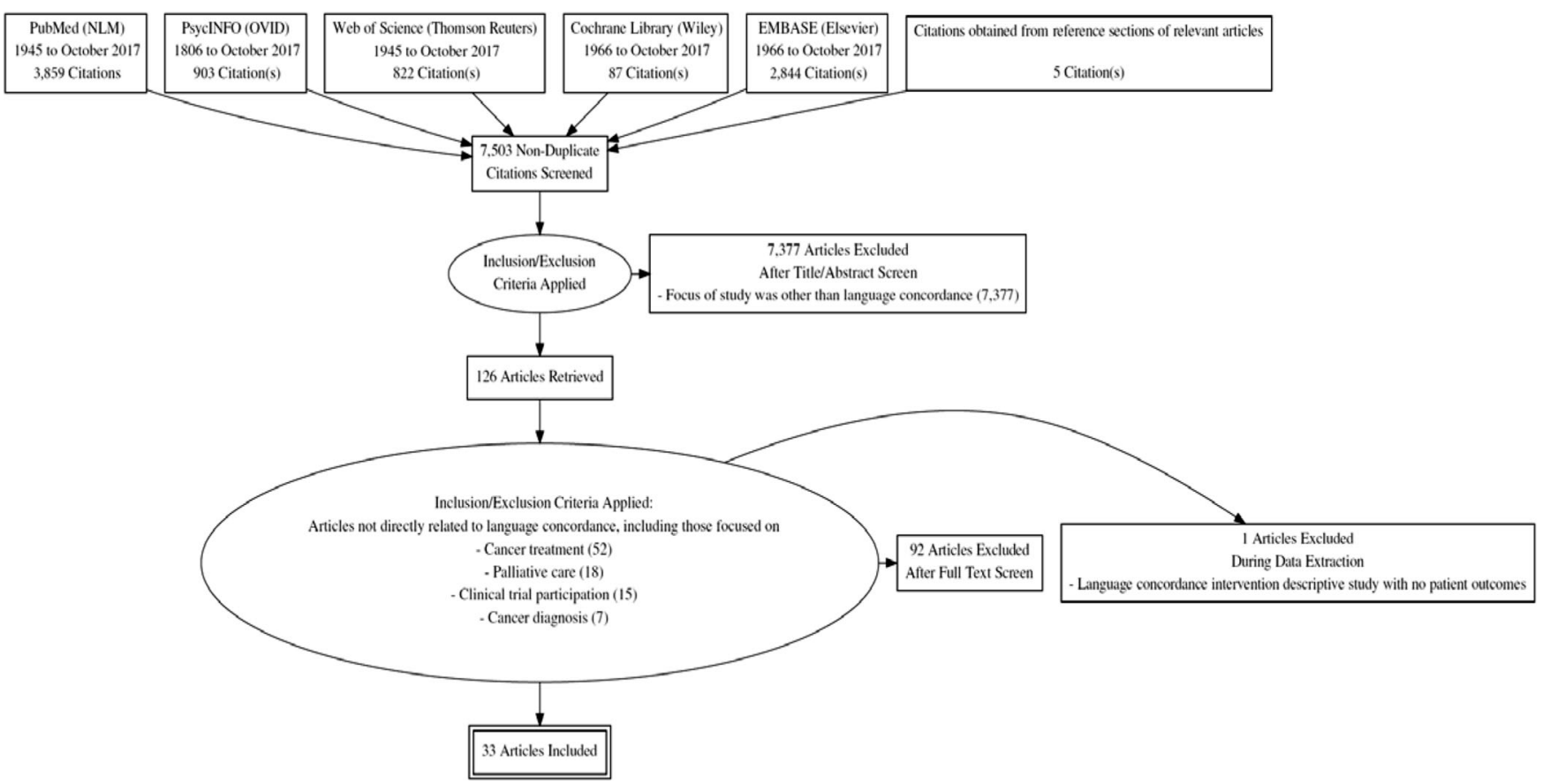

Figure 1 PRISMA flow diagram.

receiving language-concordant care, while $15 \%(5 / 33)$ of studies demonstrated no difference in outcomes, and $9 \%$ (3/33) studies demonstrated worse outcomes in patients receiving language-concordant care (Table 2).

\section{QUALITY OF CARE: PRIMARY CARE}

Among the studies looking at quality of care in a primary care setting, 4 demonstrated that language concordance had a positive impact on one or more outcomes studied ${ }^{30}, 32,33,36$ (better patient experience, higher likelihood that patients would receive and agree with counseling on diet and physical activity, and better access to and utilization of primary care providers), and 3 showed no difference ${ }^{31,35}$ (the rate of flu vaccines and mammography and reported quality of wellchild care), while 2 demonstrated a negative effect of language-concordant care on an outcome ${ }^{17,22}$ (lower colorectal screening rates in both studies).

\section{Quality of Care: Diabetes}

In the studies examining quality of care for diabetic patients, 3 showed that language concordance had a positive impact on an outcome $e^{38,39,41}$ (better glycemic control, significant reduction in LDL, blood pressure, and $\mathrm{HbA1C}$, and higher rates of adherence to CVD medications) and 1 did not show a difference in outcomes ${ }^{37}$ (in blood pressure, LDL, or HbA1C).

\section{QUALITY OF CARE: PAIN MANAGEMENT}

There was 1 study examining quality of care in pain management, ${ }^{42}$ which found that Spanish fluency and level of experience with Hispanic/Latino patients with pain had an impact on implementation of established pain management practices, while treatment with opioids was more influenced by practical matters and beliefs (e.g., finances and addiction concerns) rather than provider factors.

\section{QUALITY OF CARE: CANCER}

In the studies examining quality of care for cancer patients, 1 study showed that the language-concordant intervention was associated with higher rates of radiation therapy following surgery for breast cancer but not for prostate cancer patients ${ }^{44}$ (where there was no statistically significant difference), and another study showed that language-concordant intervention resulted in higher rates of colorectal cancer screening (more participants returned the fecal occult blood test).

\section{QUALITY OF CARE: HOSPITAL SETTING}

In the 3 studies that examined quality of care in the hospital setting, 2 showed a positive impact of language concordance $^{46,47}$ (increased satisfaction with care, fewer ED visits upon discharge, better quality transition between the hospital and the outpatient setting) and 1 showed a negative outcome ${ }^{45}$ (longer ED throughput times).

\section{Satisfaction with Care/Communication}

The 8 studies looking at satisfaction with care and communication each showed at least one positive outcome ${ }^{15}, 21,48-52$ (higher satisfaction with care, fewer questions about care, 

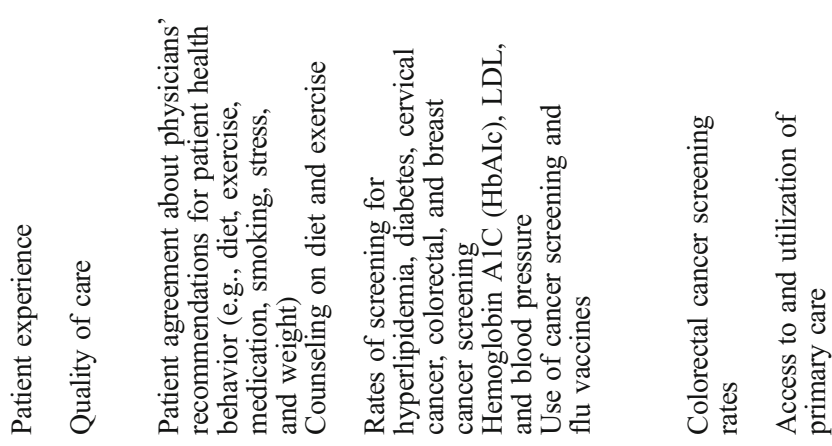

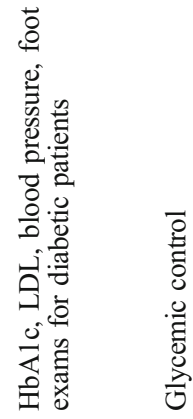
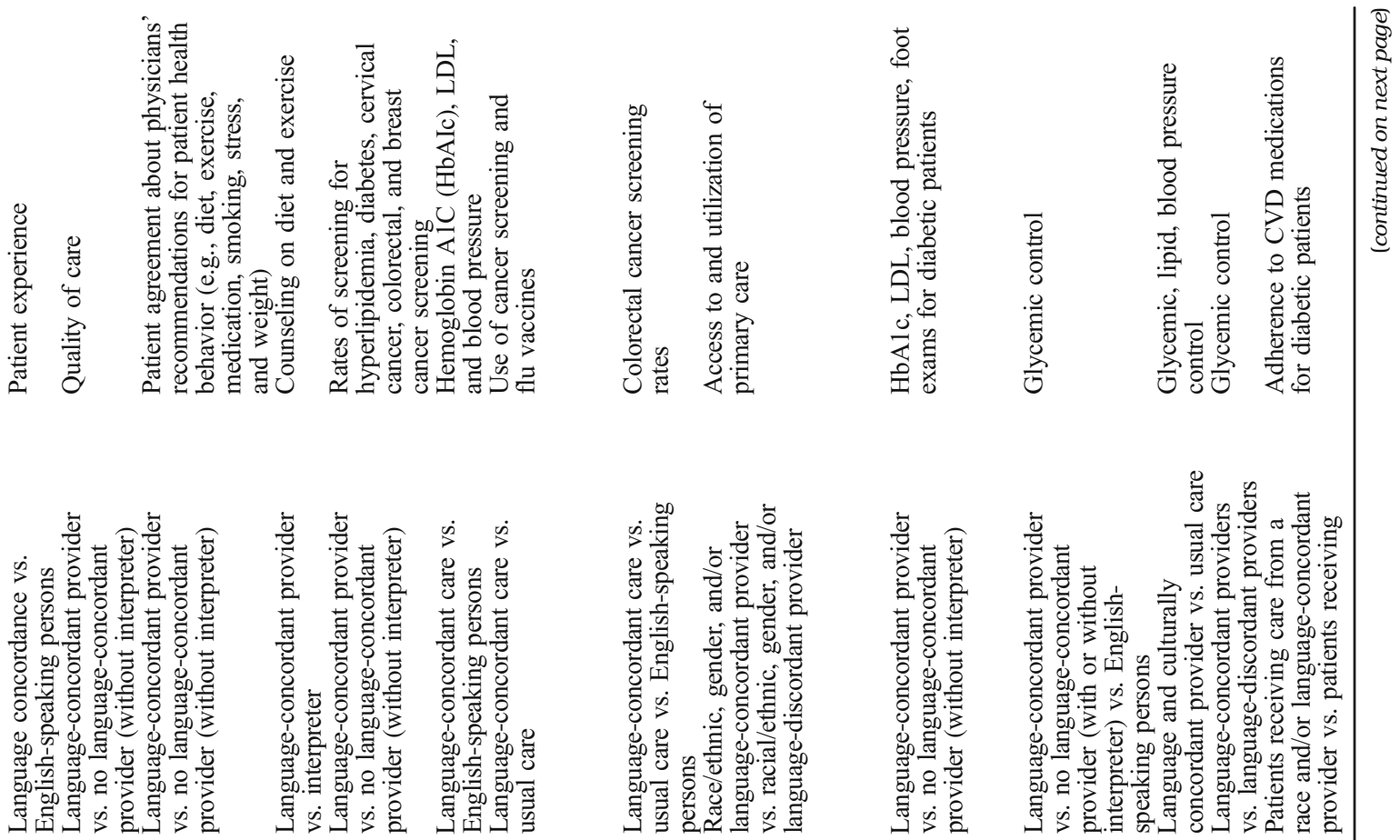

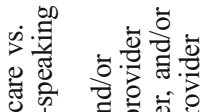
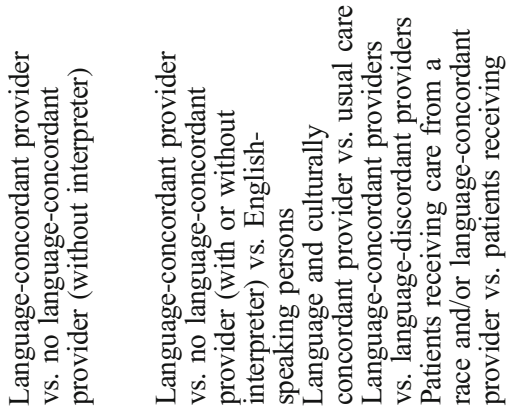

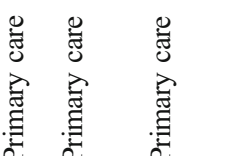

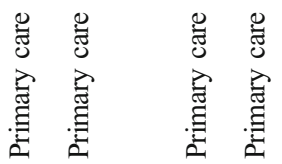

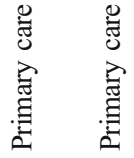

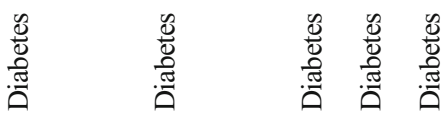

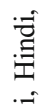

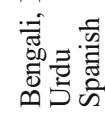

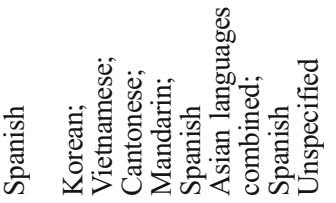

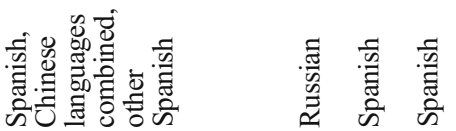

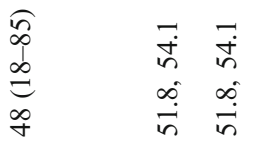

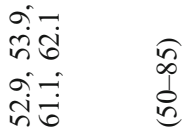

$\frac{2}{\frac{\pi}{2}}$

氧

$\left(\frac{10}{20}\right.$

$+\infty$

in $\dot{m}$

远

in $\infty \pi$ ก

in $\quad$ in

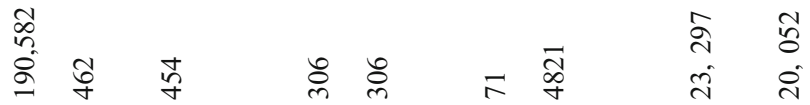

के है

if $\underset{\infty}{\infty}$ in

.

$z \quad 2$

.

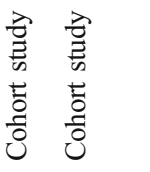

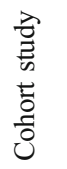

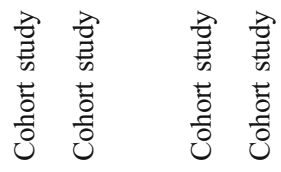

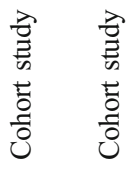

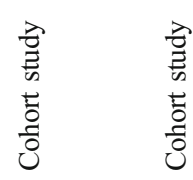

ई̊)

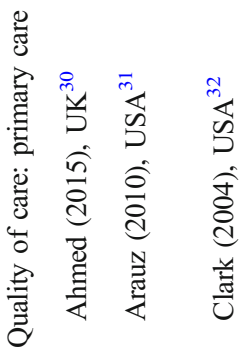

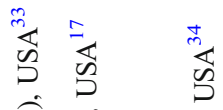

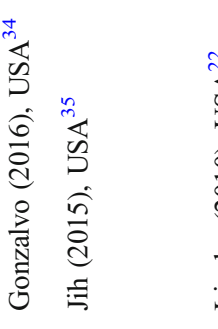

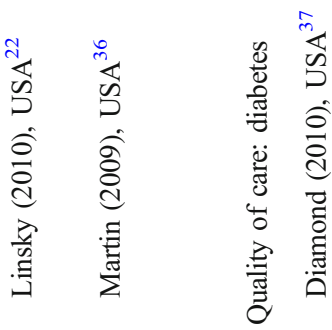

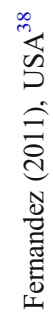

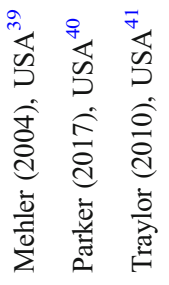




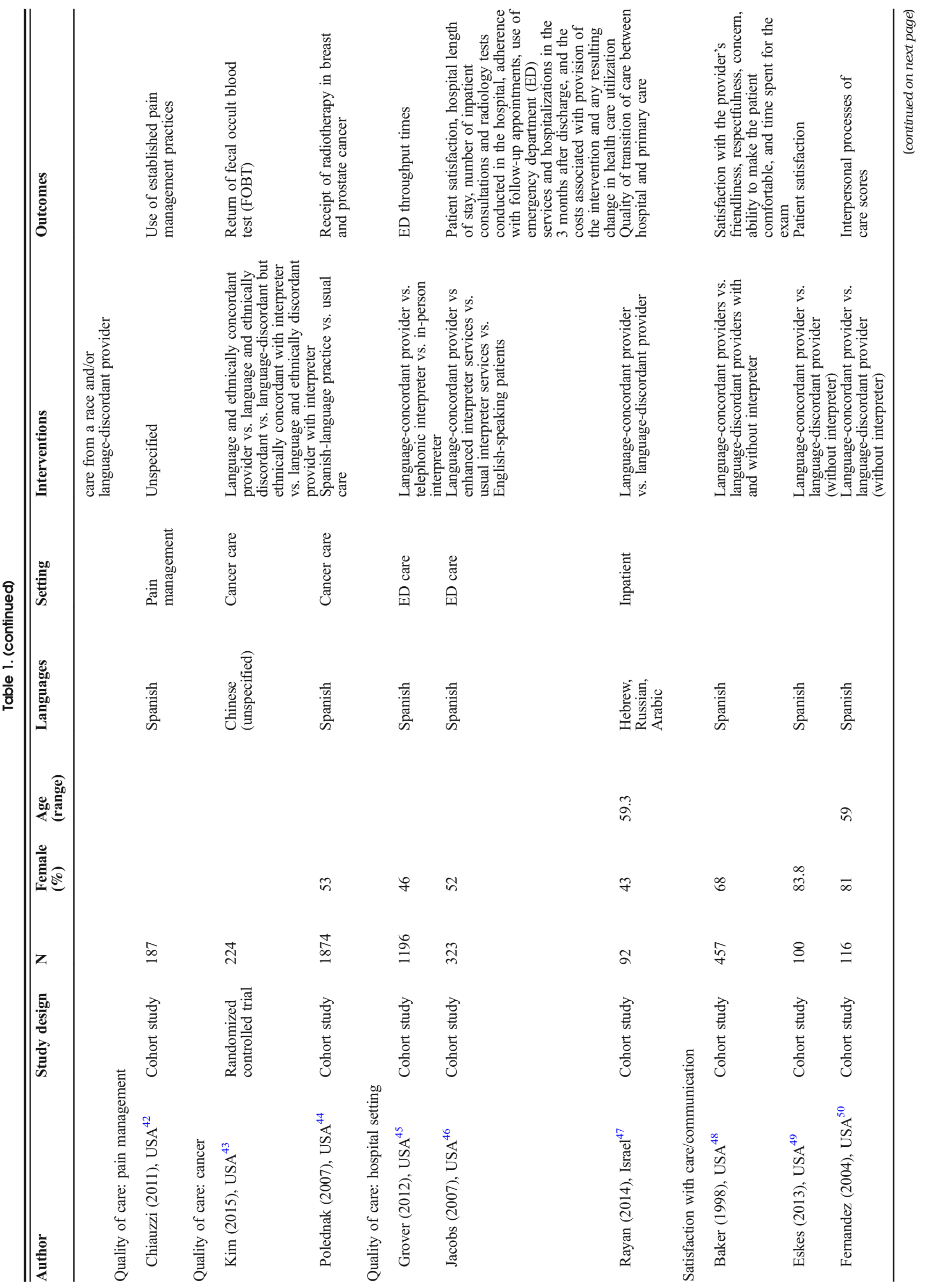




\begin{tabular}{|c|c|c|c|c|c|c|c|c|c|c|c|}
\hline 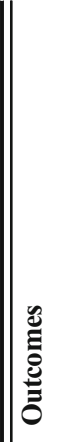 & 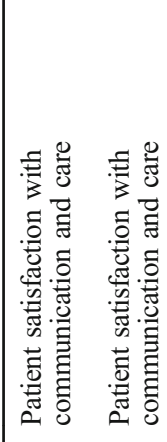 & 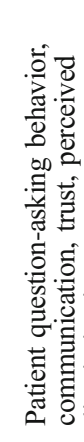 & 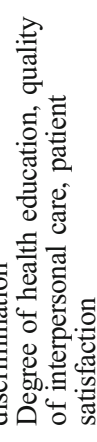 & 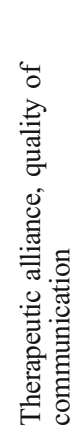 & \multicolumn{3}{|c|}{ 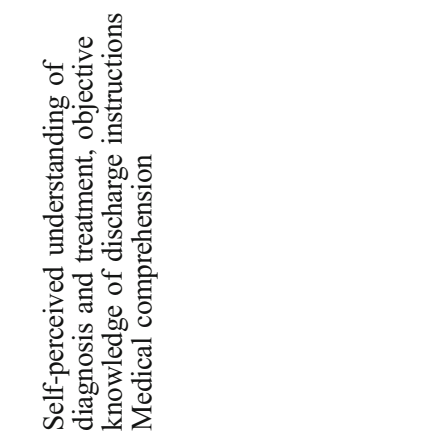 } & & \multicolumn{2}{|c|}{ 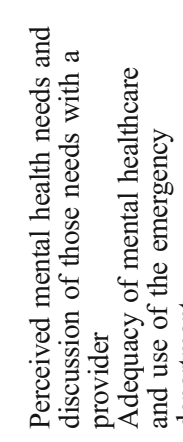 } & 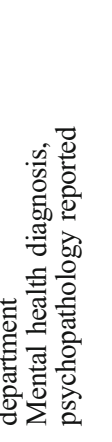 \\
\hline 产 & 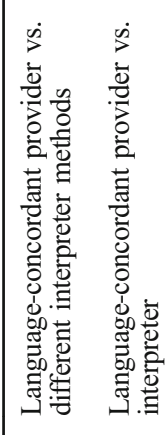 & 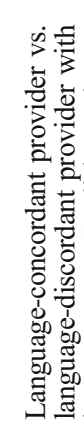 & 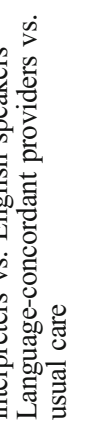 & 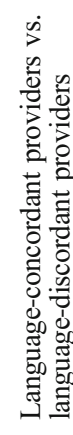 & & 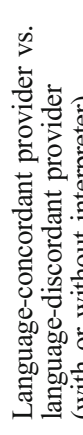 & 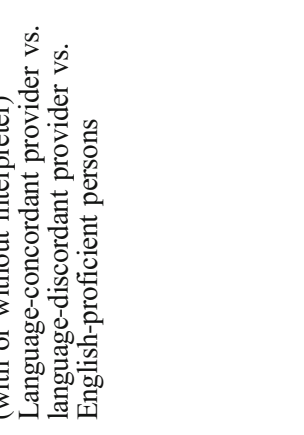 & & 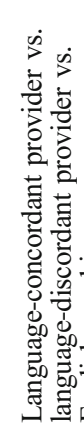 & 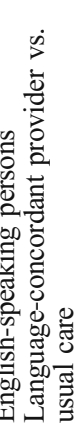 & 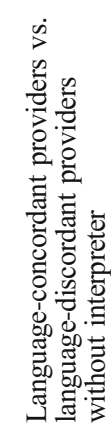 \\
\hline 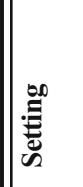 & 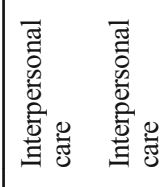 & 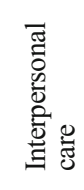 & 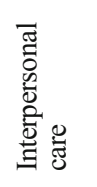 & 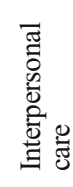 & & 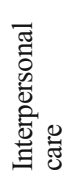 & 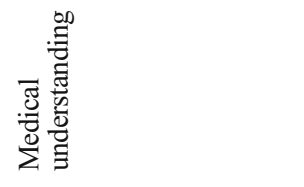 & & 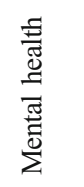 & 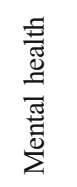 & 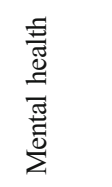 \\
\hline 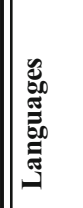 & 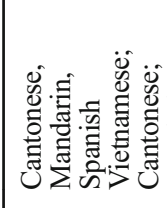 & 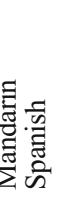 & 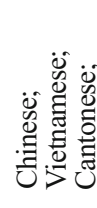 & 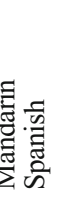 & & 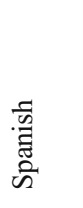 & 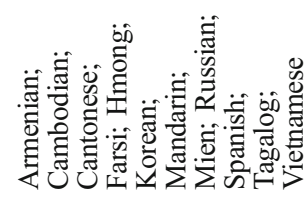 & & 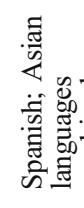 & 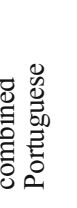 & $\begin{array}{l}\bar{v} \\
\text { 产 } \\
\text { की }\end{array}$ \\
\hline 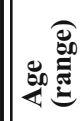 & & & & $\stackrel{n}{\ddot{\gamma}}$ & & & & & & & $\frac{\overparen{I}}{\stackrel{d}{d}}$ \\
\hline 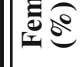 & $\Phi$ & & $\hat{\sigma}$ & $\vec{\Phi}$ & & & $\stackrel{\infty}{n}$ & & $\tilde{n}$ & $\stackrel{m}{n}$ & \& \\
\hline Z & $\stackrel{n}{\stackrel{n}{N}}$ & 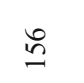 & 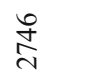 & $\stackrel{\infty}{\leftrightarrow}$ & & in & 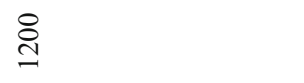 & & ¿্ঠ & $\stackrel{\infty}{\sim}$ & 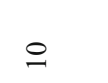 \\
\hline 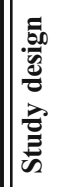 & 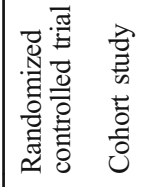 & 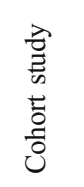 & 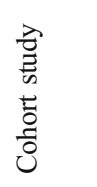 & 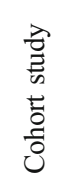 & & 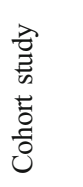 & 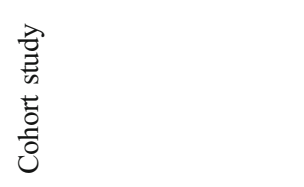 & & 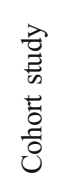 & 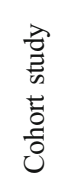 & 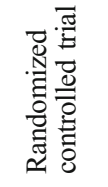 \\
\hline 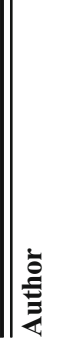 & 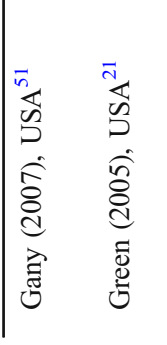 & 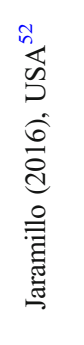 & 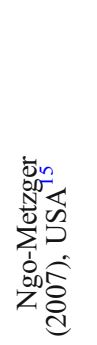 & 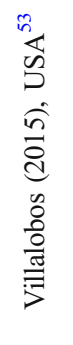 & 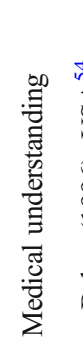 & 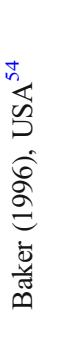 & 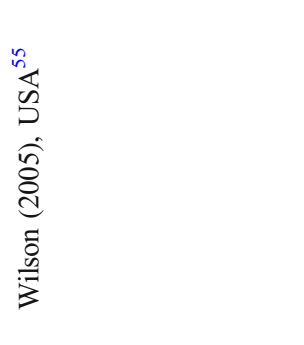 & 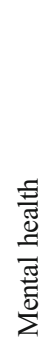 & 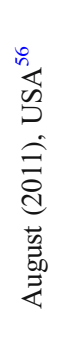 & 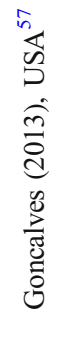 & 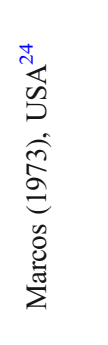 \\
\hline
\end{tabular}




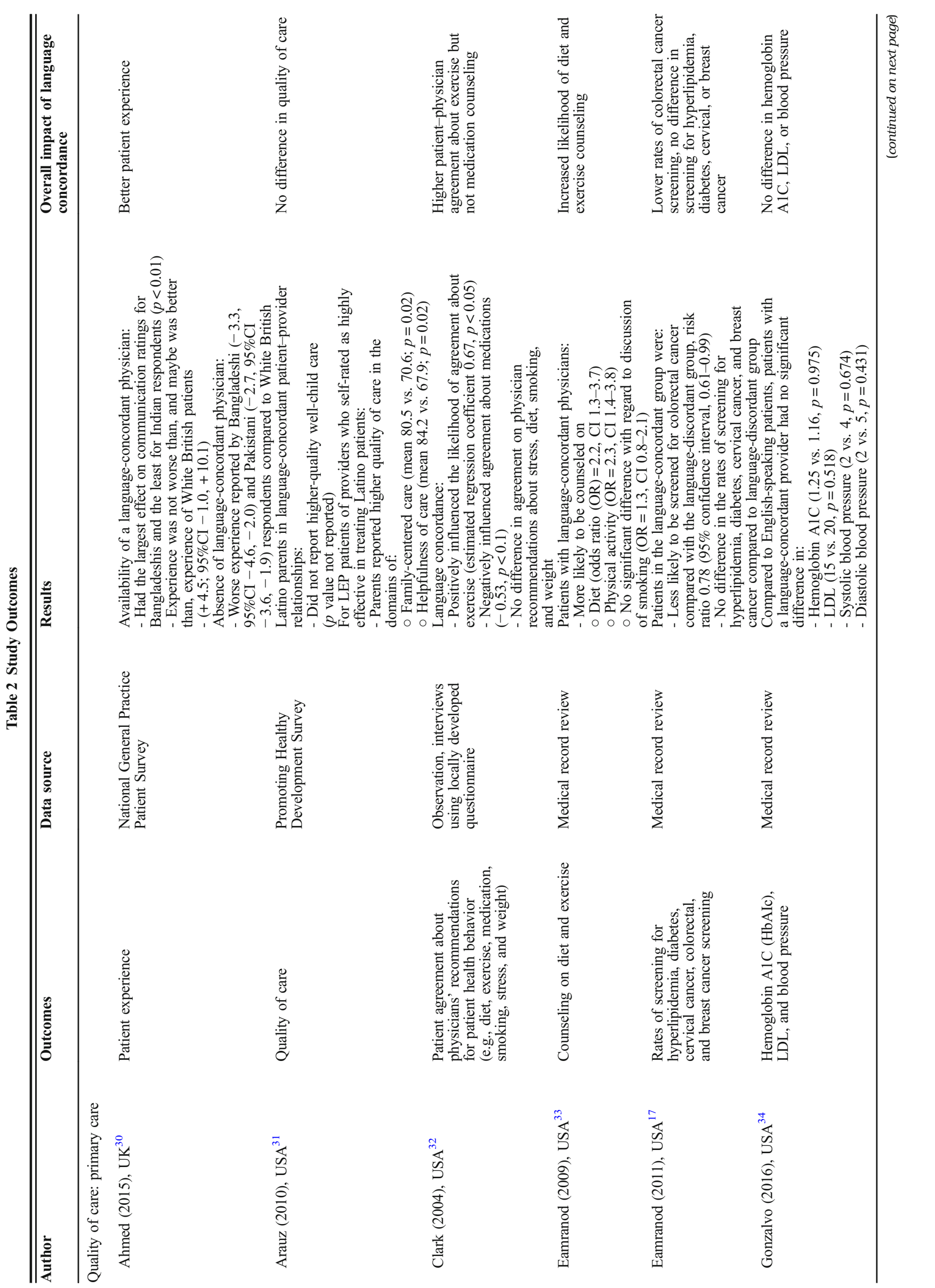




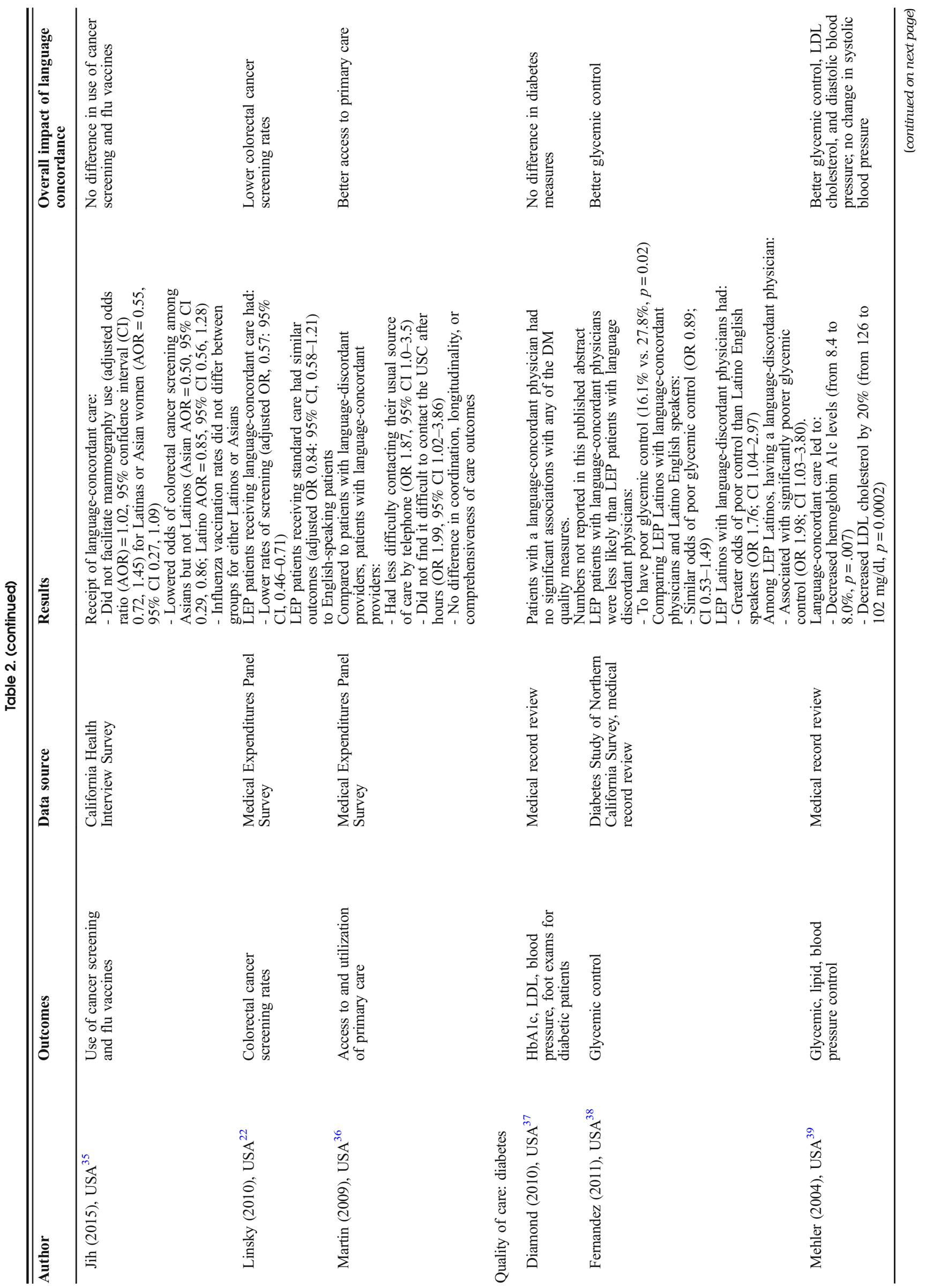




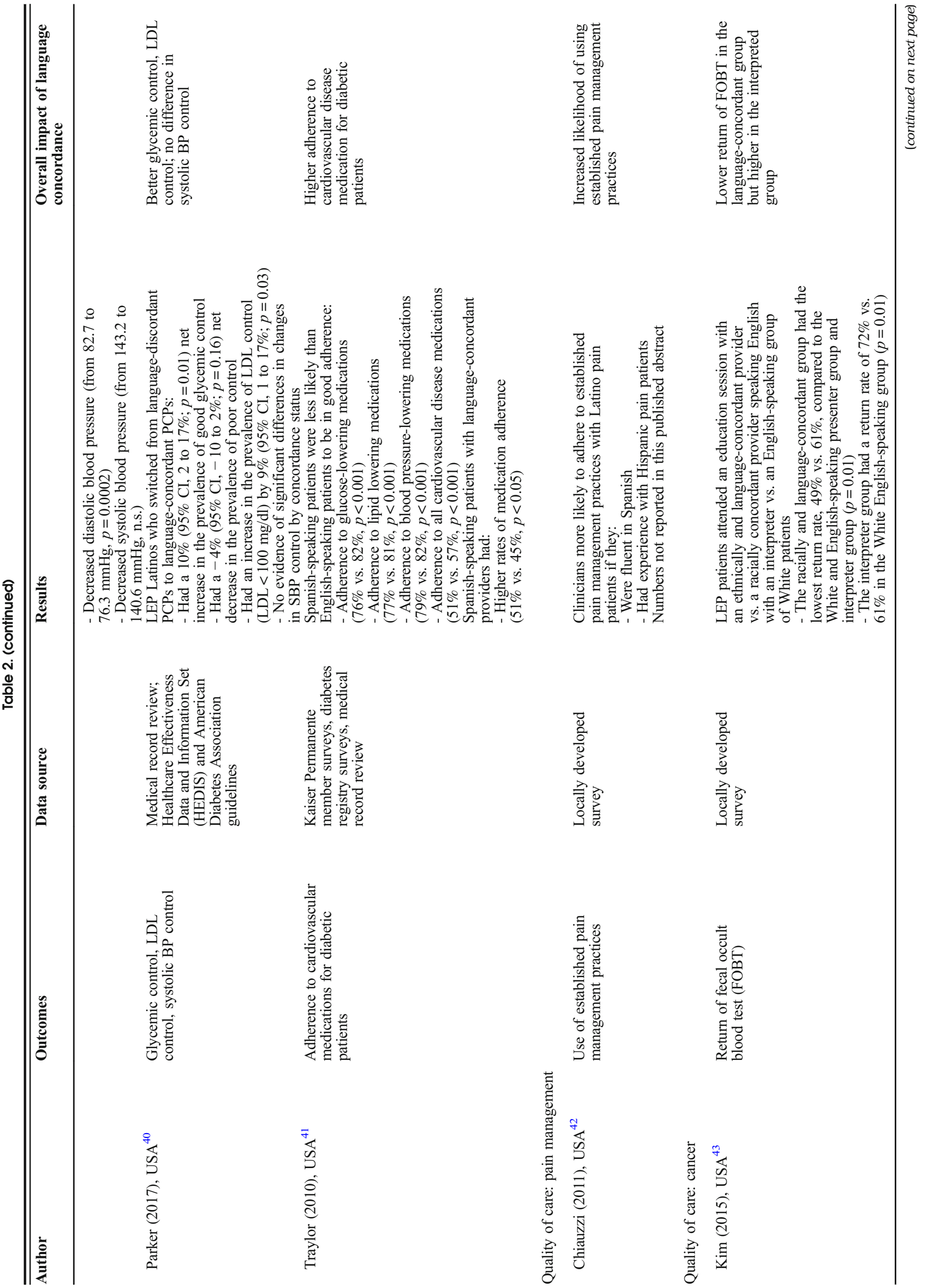




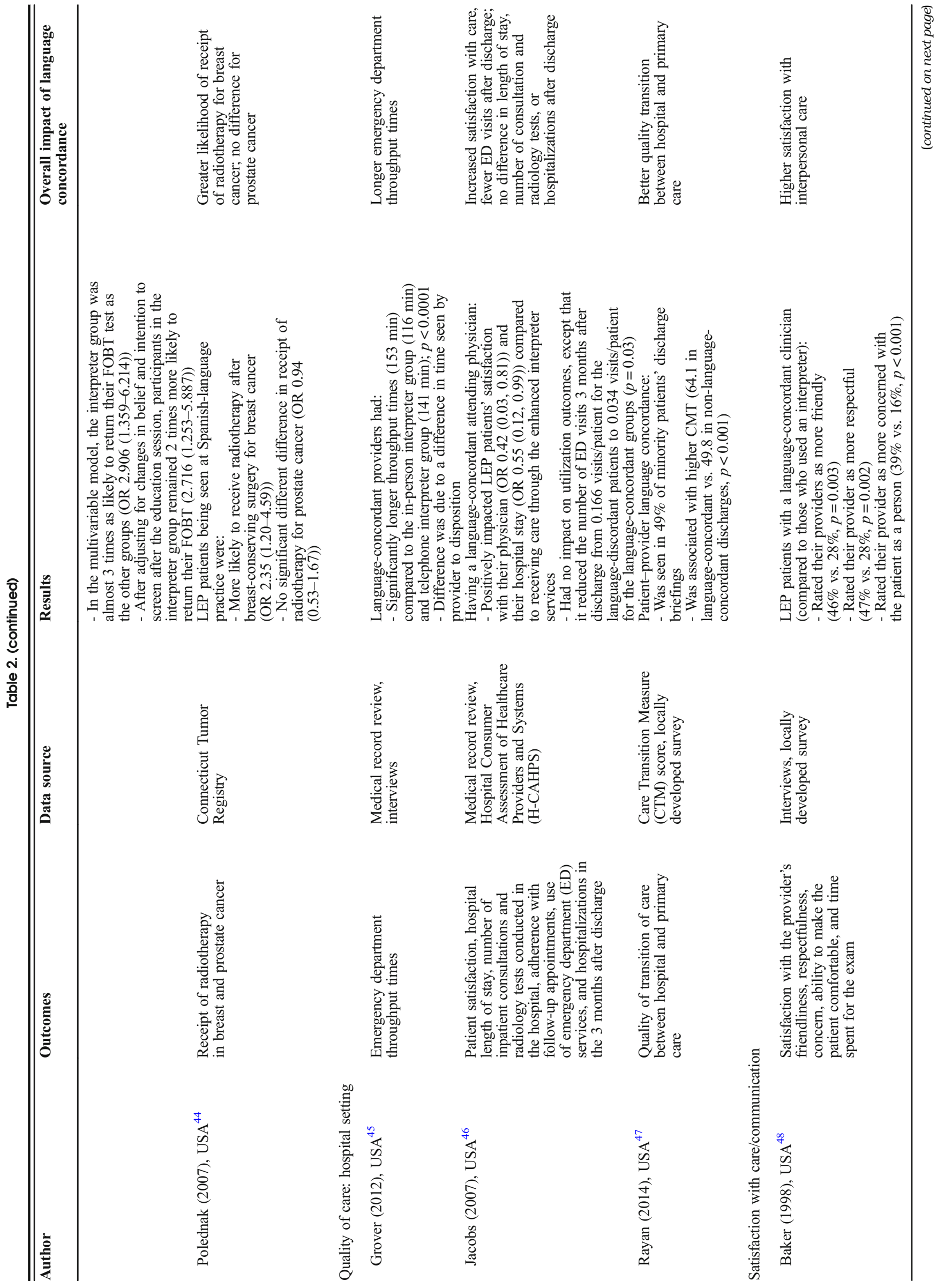




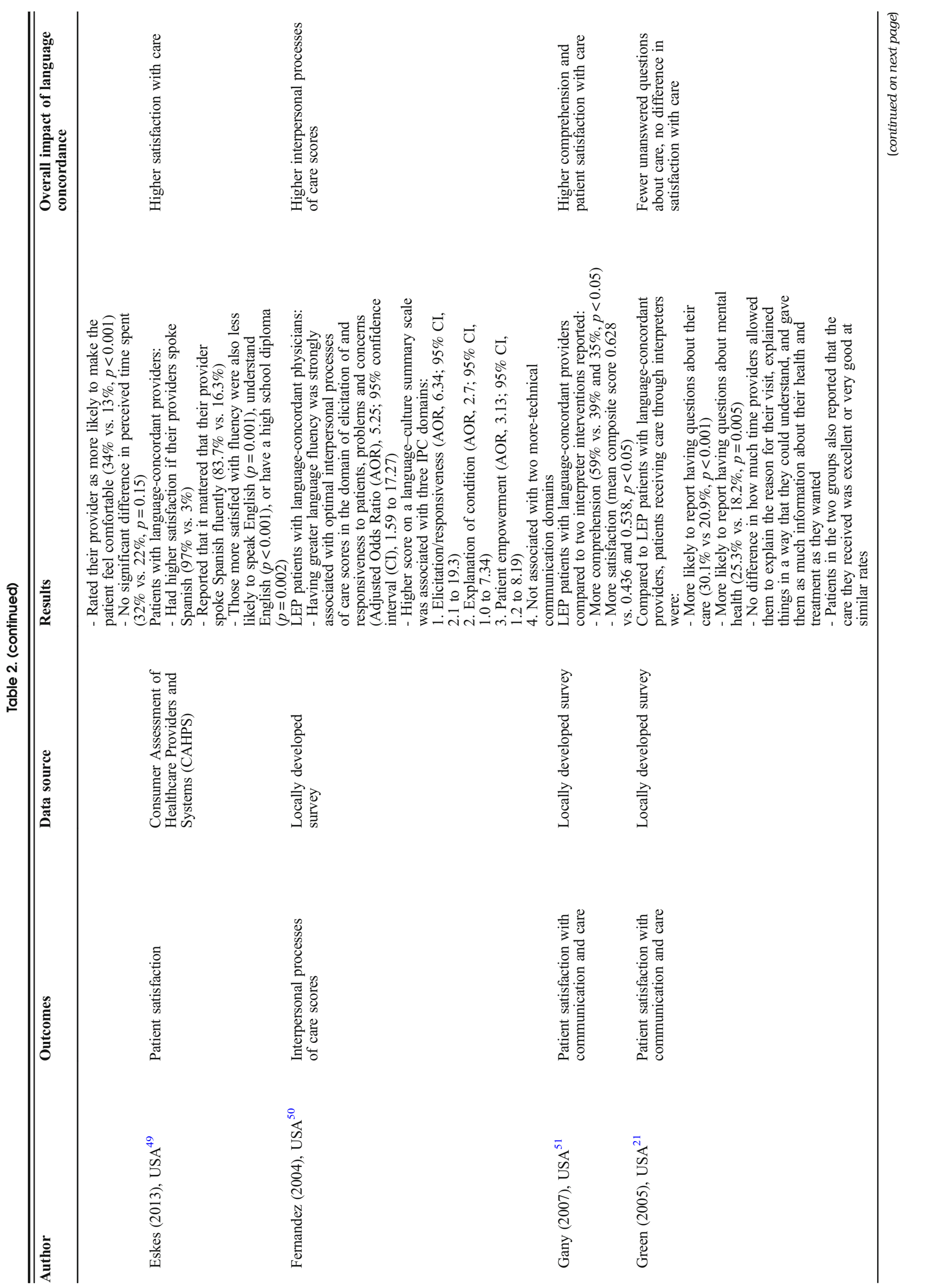




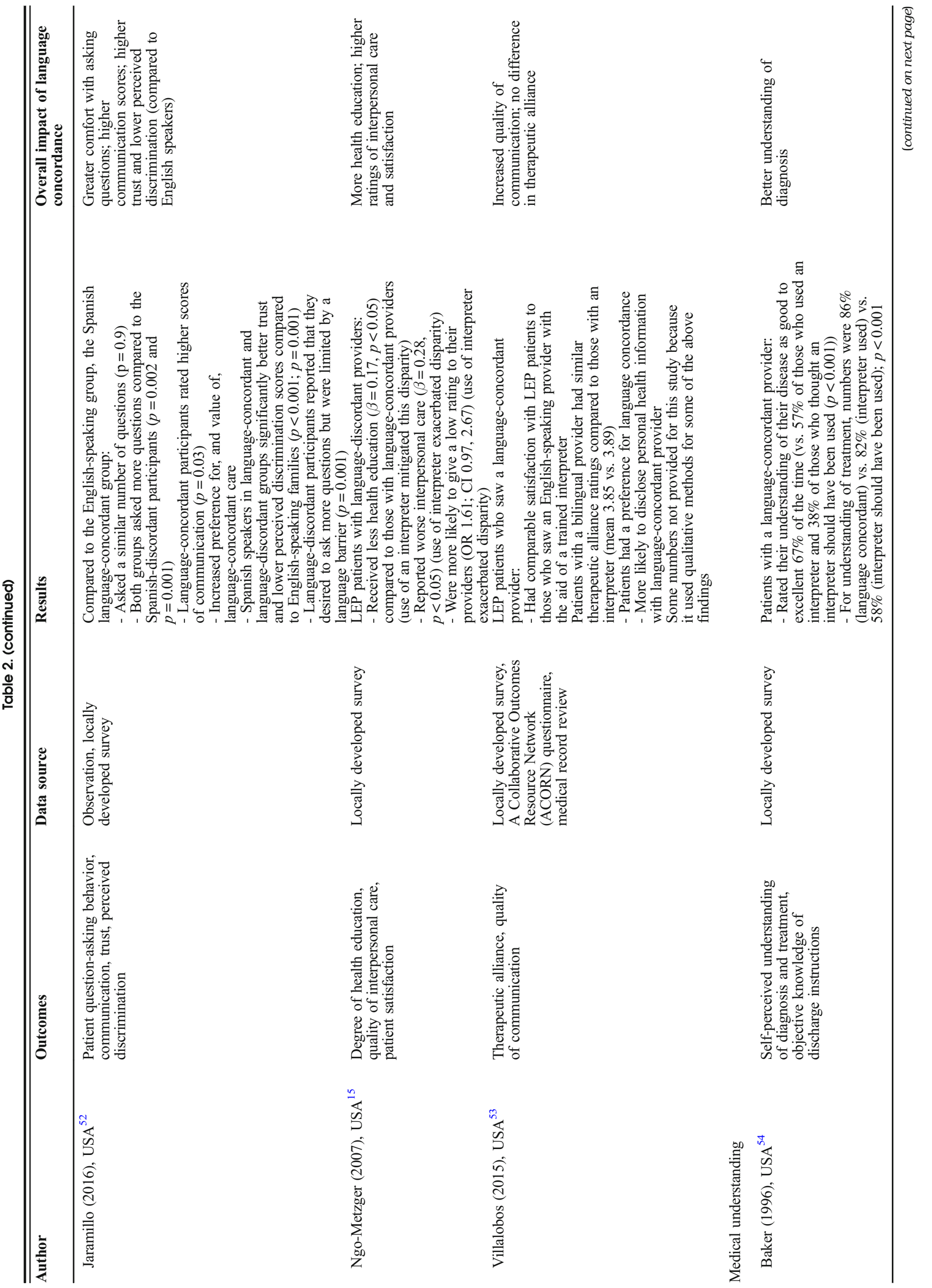




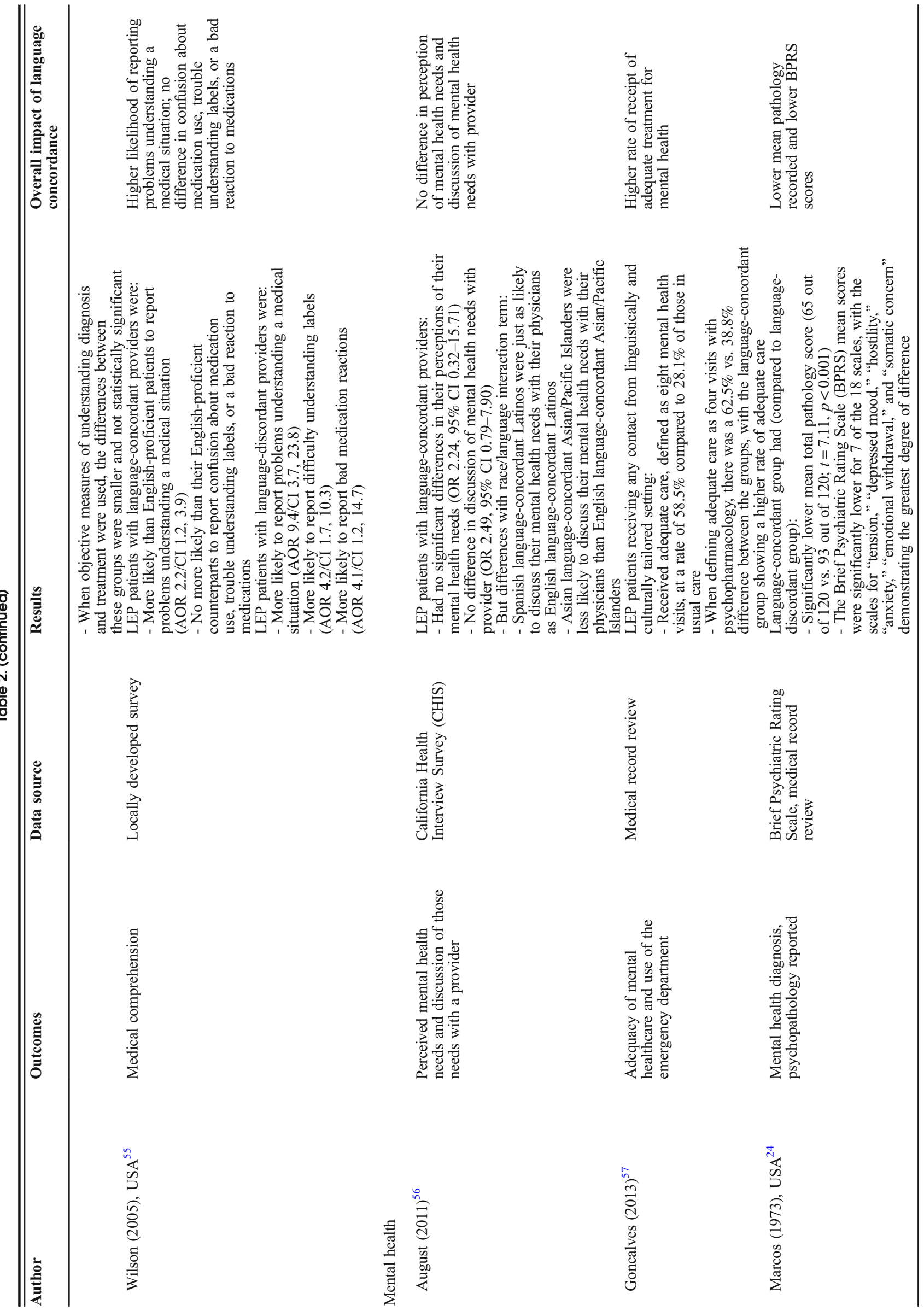


better health education, enhanced privacy, enhanced communication, and increased therapeutic alliance).

\section{Medical Understanding}

Of the 2 studies evaluating medical understanding, each showed a positive outcome ${ }^{54,55}$ (better understanding of diagnosis and higher likelihood of reporting problems understanding a medical situation in medical understanding).

\section{Mental Health}

Among the 3 studies focusing on mental health, 2 showed a positive outcome $\mathrm{e}^{24,57}$ (higher rates of adequate treatment and lower rates of pathological symptoms identified), with 1 showing no difference in the perception of mental health needs and discussion of these needs with a provider. ${ }^{56}$

\section{DISCUSSION}

Overall, the results of this review support the notion that language-concordant care is associated with better outcomes for LEP patients. Of the 33 studies included, the majority demonstrated a positive impact of language-concordant care on at least one of the major outcomes studied. The positive findings identified included patient-reported measures, such as patient satisfaction and understanding of diagnosis, ${ }^{48,49,51}$ and objective measures, including glycemic control and blood pressure for diabetic patients. ${ }^{38-40}$ These results align with our initial hypothesis that language-concordant care leads to better outcomes for LEP patients but there remain contrasting findings that may indicate some unintended consequences of language concordance.

The positive effects of language concordance are widespread, though there are some areas where receiving care from a language-concordant provider may be particularly advantageous. The majority of studies related to patient satisfaction, utilization of and access to care, and self-perceived knowledge of diagnosis demonstrated that language-concordant care had a positive impact. Additionally, the majority of publications in which process measures (blood pressure, HbA1C, and LDL) were studied showed that there is an association between language-concordant care and better process measure values for LEP patients. Taken together, these findings indicate that language-concordant care is associated with satisfaction and empowerment among patients, potentially improving their relationships with their long-term providers and producing positive health outcomes.

There were 3 studies in which language concordance was associated with negative results for LEP patients. ${ }^{17,} 22,45$ The findings of lower colorectal cancer screening rates and longer ED throughput times for patients receiving languageconcordant care may be a product of study design, or could reflect unanticipated impacts of language-concordant care that need to be taken into account by researchers and policy makers.
In the context of the studies on language-concordant care in the hospital setting, which indicates higher quality of care, better patient satisfaction, and fewer subsequent $E D$ visits for patients seen in the ED by language-concordant providers, the longer ED throughput times could be a result of providers taking more time with patients and providing better quality of care, and thus, the longer ED throughput times should not necessarily be seen as a negative outcome. The lower rates of colorectal screening that 2 studies demonstrated ${ }^{17,22}$ may be the result of better elucidation of the risks of colorectal cancer screening by language-concordant providers, which could lead some patients to opt out of the procedure based on an improved understanding. It may also reflect a patient-provider dynamic where LEP patients have more autonomy in making healthcare decisions. It is also possible that the providers considered to be language concordant in these studies were not truly fluent in their patients' preferred languages. There was no assessment of the clinicians' non-English language abilities in any of the studies demonstrating lower rates of colorectal cancer screening. These findings may reflect partial language concordance and poor quality of communication around colorectal cancer screening. The studies demonstrating longer ED throughput times and lower rates of colorectal cancer screening warrant future research to understand the underlying reasons for the findings before it is concluded that they are indicative of inferior patient care.

Although the findings of this review are consistent with previous research in demonstrating the overall positive impact of language concordance, there are limitations that must be addressed. The quality of these studies, as appraised by the Downs and Black Checklist, was generally high, which lends validity to the findings of this review. However, very few of the included studies assessed the fluency of the languageconcordant providers, a key variable impacting the relevance of each study's findings. Without this information, we are left without knowing whether the quality of communication is comparable to that of an English-speaking patient-provider dyad (which often served as the comparison group). This relates to a larger issue in the landscape of patient-provider language concordance, which is that there is currently no standardized assessment of clinician language proficiency that is consistently used by healthcare facilities. If such an assessment were used and its results were reported in future studies, this data would further help in appraising the quality of the research. Another limitation of this review is the fact that most of the studies included were performed in or near major cities in the USA and in clinics or hospitals affiliated with academic medical centers, limiting the generalizability of the findings to other countries and healthcare settings.

\section{CONCLUSIONS}

Given the rapidly growing LEP population in the USA, efforts to improve quality of and access to care will be ineffective if 
they do not attempt to address the barriers faced by LEP patients. The findings of this review indicate that, in the vast majority of situations, language-concordant care improves outcomes. Almost all of the studies included were of good quality, but none provided a standardized assessment of provider language skills. In order to systematically evaluate the impact of truly language-concordant care on outcomes and draw meaningful conclusions, future studies must include an assessment of clinician language proficiency and longitudinal observational studies. Language-concordant care offers an important way for hospitals to meet the unique needs of their LEP patients.

Corresponding Author: Lisa Diamond, MD MPH; Memorial Sloan Kettering Cancer Center, 485 Lexington Avenue, 2nd Floor, New York, NY 10017, USA (e-mail: diamond@mskcc.org).

Funding Information Dr. Diamond reported salary support from grants K07 CA184037 and P3O CA008748 from the National Cancer Institute and $A D-1409-23627$ from the Patient-Centered Outcomes Research Institute. The authors received financial support from the Memorial Sloan Kettering Cancer Center SCORE Program and Summer Medical Student Research Fellowship Program (P30 CA008748 and R25 CA020449).

\section{Compliance with Ethical Standards:}

Conflict of Interest: The authors declare that they do not have any conflict of interest.

\section{REFERENCES}

1. 2007-2011 American Community Survey 5-Year Estimates. American FactFinder: US Census Bureau. U.S. Census Bureau. 2011.

2. Ryan C. Languages in the United States: 2011. US Census Bureau. 2013.

3. LEP.gov. Commonly askd questions and answers regarding Limited English Proficent (LEP) Individuals. 2011. Available from: http://www. lep.gov/faqs/faqs.html\#One_LEP_FAQ. Accessed 15 Sept 2018

4. Fernandez A, Schillinger D, Warton EM, Adler N, Moffet H, Schenker $\mathbf{Y}$, et al. Language Barriers, Physician-Patient Language Concordance, and Glycemic Control Among Insured Latinos with Diabetes: The Diabetes Study of Northern California (DISTANCE). J Gen Intern Med. 2011;26(2):170-6.

5. Flores G, Abreu M. Tomany-Korman SC. Limited English proficiency, primary language at home, and disparities in children's health care: how language barriers are measured matters. Public Health Rep. 2005; 120(4):418.

6. Kandula NR, Lauderdale DS, Baker DW. Differences in Self-Reported Health Among Asians, Latinos, and Non-Hispanic Whites: The Role of Language and Nativity. Ann Epidemiol. 2007;17(3):191-8.

7. Timmins CL. The impact of language barriers on the health care of Latinos in the United States: a review of the literature and guidelines for practice. J Midwifery Women's Health. 2002;47(2):80-96.

8. Derose KP, Baker DW. Limited English proficiency and Latinos' use of physician services. Med Care Res Rev. 2000;57(1):76-91.

9. DuBard CA, Gizlice Z. Language Spoken and Differences in Health Status, Access to Care, and Receipt of Preventive Services Among US Hispanics. Am J Public Health. 2008;98(11):2021-8.

10. Fiscella K, Franks P, Doescher MP, Saver BG. Disparities in health care by race, ethnicity, and language among the insured: findings from a national sample. Med Care. 2002;40(1):52-9.

11. Jacobs EA, Karavolos K, Rathouz PJ, Ferris TG, Powell LH. Limited English proficiency and breast and cervical cancer screening in a multiethnic population. Am J Public Health. 2005;95(8): 1410-6.
12. Kandula NR, Wen M, Jacobs EA, Lauderdale DS. Low rates of colorectal, cervical, and breast cancer screening in Asian Americans compared with non-Hispanic whites. Cancer. 2006; 107(1):184-92.

13. Wilson E, Chen AH, Grumbach $\mathbf{K}$, Wang $\mathbf{F}$, Fernandez A. Effects of limited English proficiency and physician language on health care comprehension. J Gen Intern Med. 2005;20(9):800-6.

14. Diamond LC, Wilson-Stronks A, Jacobs EA. Do hospitals measure up to the national culturally and linguistically appropriate services standards? Med Care. 2010;48(12):1080-7.

15. Ngo-Metzger Q, Sorkin DH, Phillips RS, Greenfield S, Massagli MP, Clarridge B, et al. Providing high-quality care for limited English proficient patients: the importance of language concordance and interpreter use. J Gen Intern Med. 2007;22 Suppl 2:324-30.

16. Manson A. Language concordance as a determinant of patient compliance and emergency room use in patients with asthma. Med Care. 1988;26(12):1119-28.

17. Eamranond PP, Davis RB, Phillips RS, Wee CC. Patient-physician language concordance and primary care screening among spanishspeaking patients. Med Care. 2011;49(7):668-72.

18. Prince $\mathbf{D}$, Nelson $\mathbf{M}$. Teaching Spanish to emergency medicine residents. Acad Emerg Med. 1995;2(1):32-6; discussion 6-7.

19. Regenstein M, Andres E, Wynia MK. Appropriate use of non-Englishlanguage skills in clinical care. JAMA. 2013;309(2):145-6.

20. Diamond LC, Tuot DS, Karliner LS. The use of Spanish language skills by physicians and nurses: policy implications for teaching and testing. J Gen Intern Med. 2012;27(1):117-23.

21. Green AR, Ngo-Metzger Q, Legedza AT, Massagli MP, Phillips RS, Iezzoni LI. Interpreter services, language concordance, and health care quality. Experiences of Asian Americans with limited English proficiency. J Gen Intern Med. 2005;20(11):1050-6.

22. Linsky A, McIntosh N, Cabral H, Kazis LE. Patient-provider language concordance and colorectal cancer screening. J Gen Intern Med 2011;26(2):142-7.

23. Schardt C, Adams MB, Owens T, Keitz S, Fontelo $\mathbf{P}$. Utilization of the PICO framework to improve searching PubMed for clinical questions. BMC Med Inform Decis Mak. 2007;7(1):16

24. Marcos LR, Alpert M, Urcuyo L, Kesselman M. The effect of interview language on the evaluation of psychopathology in Spanish-American schizophrenic patients. Am J Psychiatry. 1973;130(5):549-53.

25. Moher D, Liberati A, Tetzlaff J, Altman DG. Preferred reporting items for systematic reviews and meta-analyses: the PRISMA statement. Ann Intern Med. 2009; 151(4):264-9.

26. Flores G. The impact of medical interpreter services on the quality of health care: a systematic review. Med Care Res Rev. 2005;62(3):255-99.

27. Jacobs E, Chen AH, Karliner LS, AGGER-GUPTA N, Mutha S. The need for more research on language barriers in health care: a proposed research agenda. Milbank Q. 2006;84(1):111-33.

28. Karliner LS, Jacobs EA, Chen AH, Mutha S. Do professional interpreters improve clinical care for patients with limited English proficiency? A systematic review of the literature. Health Serv Res. 2007;42(2):727-54.

29. Mehin R, Burnett R, Brasher P. Does the new generation of high-flex knee prostheses improve the post-operative range of movement? A metaanalysis. J Bone Joint Surg Br. 2010;92(10):1429-34.

30. Ahmed F, Abel GA, Lloyd CE, Burt J, Roland M. Does the availability of a South Asian language in practices improve reports of doctor-patient communication from South Asian patients? Cross sectional analysis of a national patient survey in English general practices. BMC Fam Pract. 2015;16:55

31. Arauz Boudreau AD, Fluet CF, Reuland CP, Delahaye J, Perrin JM Kuhlthau K. Associations of providers' language and cultural skills with Latino parents' perceptions of well-child care. Acad Pediatr. 2010;10(3): 172-8.

32. Clark T, Sleath B, Rubin RH. Influence of ethnicity and language concordance on physician-patient agreement about recommended changes in patient health behavior. Patient Educ Couns. 2004;53(1):8793.

33. Eamranond PP, Davis RB, Phillips RS, Wee CC. Patient-physician language concordance and lifestyle counseling among spanish-speaking patients. J Immigr Minor Health. 2009;11(6):494-8.

34. Gonzalvo JD, Sharaya NH. Language Concordance as a Determinant of Patient Outcomes in a Pharmacist-Managed Cardiovascular Risk Reduction Clinic. J Pharm Pract. 2016;29(2):103-5.

35. Jih J, Vittinghoff E, Fernandez A. Patient-physician language concordance and use of preventive care services among limited english proficient latinos and asians. Public Health Rep. 2015;130(2):134-42. 
36. Martin BC, Shi L, Ward RD. Race, gender, and language concordance in the primary care setting. Int J Health Care Qual Assur. 2009;22(4):340 52.

37. Diamond L, Chung $\mathbf{S}$, Palaniappan $\mathbf{L}$, Luft HS. The effect of patientphysician racial/ethnic and language concordance on quality of diabetes care. J Gen Intern icine. 2010;25:S402.

38. Fernandez A, Schillinger D, Warton EM, Adler N, Moffet HH, Schenker $\mathbf{Y}$, et al. Language barriers, physician-patient language concordance, and glycemic control among insured Latinos with diabetes: the Diabetes Study of Northern California (DISTANCE). J Gen Intern Med. 2011;26(2):170-6.

39. Mehler PS, Lundgren RA, Pines I, Doll K. A community study of language concordance in Russian patients with diabetes. Ethn Dis. 2004; 14(4):584-8.

40. Parker MM, Fernandez A, Moffet HH, Grant RW, Torreblanca A, Karter AJ. Association of Patient-Physician Language Concordance and Glycemic Control for Limited-English Proficiency Latinos With Type 2 Diabetes. JAMA Intern Med. 2017;177(3):380-7.

41. Traylor AH, Schmittdiel JA, Uratsu CS, Mangione CM, Subramanian U. Adherence to cardiovascular disease medications: does patientprovider race/ethnicity and language concordance matter? J Gen Intern Med. 2010;25(11):1172-7.

42. Chiauzzi E, Black RA, Frayjo K, Reznikova M, Grimes Serrano JM Zacharoff $\mathbf{K}$, et al. Health care provider perceptions of pain treatment in Hispanic patients. Pain Pract. 2011;11(3):267-77.

43. Kim K, Chandraskar E, Lam H. Colorectal cancer screening: Does racial/ethnic and language concordance matter? In: Cancer Epidemiology Biomarkers and Prevention Conference: 7th AACR Conference on the Science of Health Disparities in Racial/Ethnic Minorities and the Medically Underserved San Antonio, TX United States Conference Start: 20141109 Conference End: 20141112 Conference Publication: (varpagings) [Internet]. 2015;24(10 SUPPL. 1) (no pagination).

44. Polednak AP. Identifying newly diagnosed Hispanic cancer patients who use a physician with a Spanish-language practice, for studies of quality of cancer treatment. Cancer Detect Prev. 2007;31(3):185-90.

45. Grover A, Deakyne S, Bajaj L, Roosevelt GE. Comparison of throughput times for limited English proficiency patient visits in the emergency department between different interpreter modalities. J Immigr Minor Health / Center Minor Public Health. 2012;14(4):602-7.

46. Jacobs EA, Sadowski LS, Rathouz PJ. The impact of an enhanced interpreter service intervention on hospital costs and patient satisfaction. J Gen Intern Med. 2007;22 Suppl 2:306-11.

47. Rayan N, Admi H, Shadmi E. Transitions from hospital to community care: the role of patient-provider language concordance. Isr $\mathrm{J}$ Health Policy Res. 2014;3:24.

48. Baker DW, Hayes R, Fortier JP. Interpreter use and satisfaction with interpersonal aspects of care for Spanish-speaking patients. Med Care. 1998;36(10): 1461-70.

49. Eskes C, Salisbury H, Johannsson M, Chene Y. Patient satisfaction with language-concordant care. J Physician Assist Educ. 2013;24(3): 14-22.

50. Fernandez A, Schillinger D, Grumbach K, Rosenthal A, Stewart AL, Wang F, et al. Physician language ability and cultural competence. An exploratory study of communication with Spanish-speaking patients. J Gen Intern Med. 2004;19(2):167-74.

51. Gany F, Leng J, Shapiro E, Abramson D, Motola I, Shield DC, et al. Patient satisfaction with different interpreting methods: a randomized controlled trial. J Gen Intern Med. 2007;22 Suppl 2:312-8.

52. Jaramillo J, Snyder E, Dunlap JL, Wright R, Mendoza F, Bruzoni M. The Hispanic Clinic for Pediatric Surgery: A model to improve parentprovider communication for Hispanic pediatric surgery patients. J Pediatr Surg. 2016;51(4):670-4.

53. Villalobos BT, Bridges AJ, Anastasia EA, Ojeda CA, Rodriguez JH, Gomez D. Effects of language concordance and interpreter use on therapeutic alliance in Spanish-speaking integrated behavioral health care patients. Psychol Serv. 2016;13(1):49-59.

54. Baker DW, Parker RM, Williams MV, Coates WC, Pitkin K. Use and effectiveness of interpreters in an emergency department. J Am Med Assoc. 1996;275(10):783-8.

55. Wilson E, Chen AH, Grumbach K, Wang F, Fernandez A. Effects of limited English proficiency and physician language on health care comprehension. J Gen Intern Med. 2005;20(9):800-6.

56. August KJ, Nguyen H, Guyen NM, Sorkin DH. Language Concordance and Patient-Physician Communication Regarding Mental Health Needs. J Am Geriatr Soc. 2011;59(12):2356-62.

57. Goncalves M, Cook B, Mulvaney-Day N, Alegria M, Kinrys G. Retention in mental health care of Portuguese-speaking patients. Transcult Psychiatry. 2013;50(1):92-107.

Publisher's Note Springer Nature remains neutral with regard to jurisdictional claims in published maps and institutional affiliations. 